\title{
On the English Translation of Cultural Image Words in the Poem Chun Jiang Hua Yue Ye From the Perspective of Leech's Conceptual Meaning and Connotative Meaning
}

\author{
Peng Wang \\ Guizhou Normal University, China
}

\begin{abstract}
Semantic translation is an important link in the translation process, starting with semantic selection. Among various semantic classification systems, Leech's seven linguistic meanings are very reasonable and suitable for translation practice, research and teaching. His conceptual meaning and connotative meaning of the seven types of linguistic meanings can provide guidance for the translation of cultural image words, such as cultural image words in the two English versions of Chun Jiang Hua Yue Ye.
\end{abstract}

Index Terms - semantic translation, semantic selection, Leech, Chun Jiang Hua Yue Ye

\section{INTRODUCTION}

Chun Jiang Hua Yue Ye, a poem by ZHANG Ruo-xu, has always been regarded as one of the greatest poems of the Tang Dynasty. WEN Yi-duo once praised it as "the poem in the poem, the top of the peak". The poem attracts many readers with its unique beauty. Its translation has also attracted the attention of scholars, because it is an important part of Chinese excellent traditional culture. There are many English versions of Chun Jiang Hua Yue Ye, such as Charles Budd's The River by Night in Spring, W.J.B Fletcher's Moon Thoughts, XU Yuan-chong's The Moon over the River on a Spring Night, and ZHAO Yan-chun's Spring, River, Flower, Moon, Night.

By July 10, 2020, the author found that there are 20 journals related to the translation of Chun Jiang Hua Yue Ye on the CNKI. The numbers is very small, but their research perspectives do cover many aspects. For example, WANG Xiao-ying (2010) explores the loss of cultural meaning in XU Yuan-chong's translation of Chun Jiang Hua Yue Ye from narrative perspective. LUO Shun-jiang and WANG Song (2012) represent "Three Orientations" in three versions of French translation of the poem from Eco-translatological perspective. LÜ Zhao-fang \& LIU Jun-ping (2015) analyzes XU Yuan-chong's English version of it based on the principle of "Three Beauties". CUI Xuan (2015) researches means of textual cohesion in XU Yuanchong's The Moon over the River on a Spring Night. ZHANG Tong-tong (2016) compares XU Yuan-chong's and TANG Yi-he's English versions of the poem to demonstrate the practicality of "Relevance Theory" in poetry translation. CHENG Fang-xia \& XU Wen-ting (2018) analyzes the original text and different translations of the Tang poem from the perspective of Halliday's theme and rheme. TANG Jun \& HE Ting-ting (2019) generalizes structural metaphor, orientational metaphor and ontological metaphor used in the translation of Chun Jiang Hиа Yue Ye.

At present, there are only three relevant studies on the translation of cultural words in Chun Jiang Hua Yue Ye. REN Jing (2015) discusses the differences of cultural vocabulary in the process of translation from the perspective of translation features. From the perspective of cognitive metaphor, SUN Xiao-yan (2018) represents noun metaphor in three English versions of this poem. Based on Nida's functional equivalence theory, YAN Ci (2020) analyzes ZHAO Yan-chun's translation and puts forward that the culture-loaded words can be translated through literal translation, free translation, and transliteration plus annotation. Therefore, in this paper, the writer attempts to explore the translation of cultural image words in XU Yuan-chong's and ZHAO Yan-chun's English translation of Chun Jiang Hua Yue Ye from the perspective of Leech's conceptual meaning and connotative meaning.

\section{Semantics AND TRANSLATION}

Translation is closely related to semantics, because semantic selection is very important in the translation process. To eliminate cultural differences, to convey same feelings of the poem, and to better improve communication, translators must choose the best proper words in translating, especially in the translation of culturally-loaded words and culturally image words.

\section{A. The Concept of Semantics}

Semantics is, by definition, "the study of meaning in language" (Hurford et al., 2007, p. 1). It is a branch of 
linguistics. And it studies the meaning of words and changes of words meaning, focusing on exploring the reasons and principles of the changes of words meaning from the social and historical perspectives (SHI Bai-li, 2005). Semantics has a long history and its origin can be traced back to the ancient Greek philosopher Socrates (LI Xiang-de \& HUA Han-jun, 1990). However, semantics did not develop into an independent science until the end of the 19th century.

In the 17th century, the adjective semantick appeared in the form of "Semantick Philosophy" in A Discourse Concerning Prodigies, which was written by John Spencer and published in 1663, and then the second edition was re-written completely by him in 1665 . Read represents that "the phrase 'Semantick Philosophy' refers to the study of the various types of divination, or, in a more up-to-date terminology, to prediction of the future on the basis of signs" (Read, 2015 , p. 78). However, the active usage of semantics did not begin until late in the 19th century, and was coined from the French linguist Michel Bréal. In his article in 1883, Bréal defined the French word "sémantique" as "the science of significations" (Riemer, 2016, p. 51). And then another French linguist, Arsène Darmesteter, adopted Bréal's term in a study of word history two years later. In 1894, an American scholar Charles R. Lanman declared: "The doctrine of the principles that underlie the processes of the development of the meanings of words may be called semantics or semasiology." In 1897, the word received a great push when Bréal's full book Semantics: Studies in the Science of Meaning was published, which was considered as a milestone in language study. In her English version in 1900, the translator Mrs. Henry Cust used the form 'semantics' without apology. Therefore, the word semantics gradually became popular among linguists until it became a universally accepted term and was more popular than semantics (Read, 2015).

\section{B. The Development Stages of Semantics}

The development stages of semantics are divided into different stages. Among them, JIA Yande (1992) thinks that semantics studies can be roughly divided into three stages: from the stage of philology to traditional semantics and then to modern semantics.

The stage of philology mainly focused on annotation of ancient books, which began in the 3rd century BC in Europe. This period has compiled and accumulated a complete set of paraphrasing methods, thus it is of important value for the studies of semantics. However, because semantic studies is not an independent system, but only pays attention to the ancient written language, and its purpose was to annotate ancient languages, so no theory has been put forward in the semantic field.

From the beginning of the 19th century to the 1920s, the semantics of this period belonged to the traditional semantics. At the beginning of the 19th century, the establishment of Saussure's general linguistics theory made language research an independent science with its own theories and methods. It is precisely in the context of the development of language research that semantics studies can become a branch of linguistics. German linguist Trier proposed the famous 'semantic field' theory to study the system of vocabulary and word meaning in language and to study their relationship (WANG Shuang-mei, 2009). It is a major leap from philology to traditional semantics. The studies of words meaning is no longer just used as a tool to understand ancient classics and customs. Its object and scope have been expanded. However, the research in this period is still not systematic, and the research scope is limited to the meaning of words.

From the 1920 s to the present is the period of modern semantics. With the rise and development of modern information technology, which is extremely closely related to language, the research of meaning has risen to an unprecedented important position. The studies of semantics therefore has been comprehensively developed and made important breakthroughs. Many schools of semantics have emerged, such as structural semantics, interpretative semantics, and generative semantics. The research of meaning of modern semantics is mostly influenced by language philosophy and cognitive linguistics on its words level, while the research on the sentence level is largely influenced by logical semantics (WANG Shuang-mei, 2009). Modern semantics uses the sememe analysis method to reach the micro level of semantics research; from word meaning as the center content to the meaning of sentences and texts; and research methods introduce logical concepts such as presupposition, proposition, and implication (JIA Yan-de, 1992). In short, the development of semantics has broken through the relatively single research level and research method of traditional semantics, showing the characteristics of interdisciplinary, multi-dimensional and multi-level.

\section{The Relationship Between Semantics and Translation}

The purpose of translation is to understand the original text and express it accurately in the target language. In briefly, the translation process includes two stages: understanding and expression, both of which involve grasping and understanding the meanings. First of all, in the stage of understanding, the translator must thoroughly understand the original text, which is a process of analyzing the meanings of the original text. Secondly, in the stage of expression, the translator should express his or her understanding of the source text in the target language. At this time, what are the intrinsic meanings of the words and sentences chosen by the translator, and whether the meanings of the original text can be accurately and appropriately reflected in the translated language, is a crucial issue.

The meaning of language consists of two parts: semantic meaning and pragmatic meaning. Semantic meaning is the static meaning of expressions, which is inherent in the language system and independent of the language environment and language users (speakers/writers, listeners/readers). Pragmatic meaning refers to the dynamic meaning of discourse, which was generated on the basis of sentence meaning and was varied with the different of language environment and language users (ZENG Xian-cai, 1993). Engene A. Nida once said: "Translating means translating the meaning." 
(ZHANG Jing-hao, 2000, p. 30) From this it is natural to say translation means to translate both semantic meaning and pragmatic meaning. Therefore, there is a close relationship between semantics and translation.

In view of the importance of semantics in the translation process, it is necessary to make further research of semantics in order to better understand and convey meaning in translation. WANG Dong-feng (2005) believes that the seven types of meaning proposed by the famous British linguist Leech is a very reasonable system, which is more suitable for translation practice, translation studies and translation teaching. The author agrees very much with this point. In this paper, the author intends to take Leech's conceptual meaning and connotative meaning as a guide to analyze how to make appropriate semantic choices when translating cultural image words of Chinese classical poetry.

\section{SEVEn TyPes OF MEANING By GEOFFREY LEECH}

Up to now, scholars have classified semantics differently. For example, in the article Meaning published in 1957, Grice distinguished between natural sense and non-natural sense. And from the perspective of language use, he divided linguistic meaning into fixed meaning, applied fixed meaning, situational meaning and speaker's situational meaning (HAN Zhong-qian, 2009). Morris divides linguistic meaning into referential meaning, linguistic meaning and pragmatic meaning in his modern semiotics theory (HE Yuan-xiu, 2009). Geoffrey Leech originally divided linguistic meaning into seven types in his Semantics in 1974: conceptual meaning, connotative meaning, stylistic meaning, affective meaning, reflected meaning, and collocative meaning and thematic meaning. Meanwhile, the seven types meaning are generalized into three broad types: conceptual meaning, associative meaning and thematic meaning (Leech, 1974). Leech's research on the classification of linguistic meaning has an impact on the linguistics community, and has effectively promoted the development of semantics.

\section{A. Conceptual Meaning}

Conceptual meaning refers to the generalization or reflection of the objective world. It is generally fixed in a dictionary and is the basic meaning of a word. Leech believes that conceptual meaning is the core of word meaning and is about the meaning of logic, cognition, and denotation. Conceptual meaning is concerned with the connection between a word and what it refers to. The word expresses what it refers to through concepts (WU Qian-guang, 1992). For example, “woman” means “妇女”, “女人”, “成年女子”.

\section{B. Connotative Meaning}

Connotative meaning is the meaning attached to the conceptual meaning, which conveys meaning through what language refers to. Connotative meaning is implicit and needs to be obtained through association on the basis of conceptual meaning. It is the supplement and extension of conceptual meaning (WU Qian-guang, 1992). For example, "women" were oppressed and discriminated against in feudal society, thus the word gained some additional connotations: "frail", "prone to tears", "emotional", and "inconstant", etc. Of course, the word woman has also gained some good connotations: "gentle", "compassionate", and "hard-working", etc.

Leech compared connotative meaning with conceptual meaning, and believed that connotative meaning has the characteristics of instability, which will change with the change of culture, time, society, and personal experience (LI Shan, 2019). In addition, because verbal communicators have different understandings of the subjective and objective knowledge referred to by words, different people will have different understandings of the connotation of the same word.

\section{Stylistic Meaning}

Stylistic meaning can express the meaning of the social environment where language is used. Leech stated that the actual use of language can be divided into different levels according to different contexts, such as formal and literary, colloquial, familiar, and slang. And the stylistic meaning of language is expressed due to those contexts (WU Qian-guang, 1992). For example, the conceptual meaning of “马” is horse, it is a general term. However, its stylistic meanings are different, "steed" is used in poems, "nag" is slang, and "gee-gee" is a childrenese.

\section{Affective Meaning}

Affective meaning is a kind of meaning which can express the emotion and attitude of the speaker. It is independent but often depends on other types of meaning (conceptual meaning, connotative meaning or stylistic meaning), or manifested by means of tone, intonation, and interjection etc. And it is most closely related to the expression function of the discourse (WU Qian-guang, 1992).

\section{E. Reflected Meaning}

Reflective meaning is the meaning conveyed by associating with another meaning of the same word. It's as if the light hits one point on the mirror and reflects to another point. In other words, because of the role of context, when expressing a certain meaning of a word, it can also make the reader/listener think of another meaning of the word. The puns created by writers in literary works, as well as taboos and euphemisms in every culture, are often related to the mapping of meaning (WU Qian-guang, 1992). For example, the word "intercourse" is related to "communication". However, people tend to say "human language is a tool of social communication" instead of saying that "human 
language is a tool of social intercourse". This is because "intercourse" is also related to "sex".

\section{F. Collocative Meaning}

Collocation meaning is the meaning produced by the habit of collocation of words. Due to the effect of language habits, in different language systems, the same word will have different collocation habits, and when the same word is matched with different words, it will also cause people to have different collocation associations and convey different messages (WU Qian-guang, 1992). For example, "pretty" and "handsome" are the same in their basic meaning, but their collocation are different, "pretty" can be used to describe "girl", "boy", "woman", "flower", "garden" or "village", while "handsome" are allocated with "boy", "man", "car", "vessel”, "overcoat", etc.

\section{G. Thematic Meaning}

Thematic meaning is the meaning conveyed by the way information is organized. The theme can be better highlighted by means of word order, arrangement of focal points, and means of emphasis. For example, Leech pointed out that "Mrs. Smith donated the first prize" is different to "The first prize was donated the first prize", for the former is to answer "What did Mrs. Smith donate" and the latter is to respond to "Who donated the first prize" (WU Qian-guang, 1992: 145-146).

\section{Application Conceptual Meaning and Connotative Meaning to the Translation of Cultural Image WORDS IN CHUN JIANG HUA YUE YE}

Conceptual Meaning and Connotative Meaning of Leech's seven types of meaning are of great significance for translation, especially the semantic choices in the translation process. These two meanings almost cover the connotation of culturally image words in Chinese classical poetry, and therefore lead to different translation strategies.

\section{A. An Introduction to the Poem Chun Jiang Hua Yue Ye and Its Author}

With "spring", "river", "flowers", "moon" and "night" as background, the poem depicts the magnificent scenery in nature, expresses the sincere and moving sentiments of parting, and praises the pure love of mankind. Taking the moon as the main line, the whole poem describes the river, the sand beach, the night sky, the field and so on under the moonlight, and then shows us a picture full of life philosophy and life fun. The rhyme and rhythm are pleasing to the ears and they change with the sentimental ups and downs of the poem.

The author of this poem is ZHANG Ruo-xu, a famous poet in the Tang Dynasty, who is disgusted with etiquette and customs in feudal society and pursues freedom, leisure and comfort in life. His poetic style was deeply influenced by the poetic style of Six Dynasties, but from the perspective of philosophical expression and artistic means, he has inherited many characteristics of Tang poetry.

\section{B. Application Conceptual Meaning to the Translation of Cultural Image Words in Chun Jiang Hua Yue Ye}

Chun Jiang Hua Yue Ye takes "moon" as its central image, closely following the five characters of "spring", "river", "flower", "moon" and "night". It combines many images such as river, beach, sky, weald, maple, frost, snow, white clouds, mirrors, canoe, swan geese, lonely women, wanderer and many other images, forming a complete and orderly poetic image group. In this image group, the river and the bright moon are the central images, and the canoe, lad and jade curtains are strung into the poem, and various human feelings such as lovesickness, melancholy and attachment are injected into the poem. The "river" and "moon" at different angles in the distance_—near and far_-give people endless reverie. The poet's emotions are realized through the orderly arrangement of images. The rivers and beaches are from far to near; the sky and the field are from high to low; the maple trees, flower forests and white clouds go from low to high. Canoe, lad, mirror platforms, swan geese, fish, dragons, thinking women, wanderer, etc., are the images imagined by the poets or virtual scenes. The combination of these images, from reality to imagination, from objects to people, from scenes to lyricism, not only conforms to the law of human thinking, but also conforms to the law of vision, forming a beautiful, harmonious and stimulating mood (LI Qing-yun, 2013).

《春江花月夜》

XU Yuan-chong: The Moon over the River on a Spring Night

ZHAO Yan-chun: Spring, River, Flower, Moon, Night

The title of this poem is controversial, because some scholars only regard it as a poem title, while others believe that the five Chinese characters are five image words. XU's and ZHAO's English version are actually the two kinds of thinking, and the writer here prefers to XU's version. Chun Jiang Hua Yue Ye was originally the title of the song. When writing songs at that time, there were lyrics after songs, which were called writing lyrics. The song Chun Jiang Hua Yue $Y e$ is to express the love of men and women. The lyrics of the song of the same name were written by CHEN Shu-bao and Emperor Sui Yang. ZHANG Ruo-xu also wrote about the feelings of young women in the boudoir as usual with half of the space (YI Zhong-tian, 2018).

江流宛转绕芳甸, 月照花林皆似霰。

XU Yuan-chong: The river winds around the fragrant islet were

The booming flowers in her light all look like snow. 
ZHAO Yan-chun: The river winds across a fragrant mead;

The moon snows the blooms with her snowy light.

Both translators translated the two words literally. However, “芳甸” means weald with lush grass, not an island. So the writer thinks that ZHAO's version is more appropriate.

空里流霜不觉飞, 汀上白沙看不见。

XU Yuan-chong: You cannot tell her beams from hoar frost in the air,

Nor from white sand upon Farewell Beach below.

ZHAO Yan-chun: Of hoarfrost in the air one takes no heed,

And on the shoal you fail to see sand white.

“汀” means shoal here. It is also the Farewell Beach of the man and the woman in this poem.

白云一片去悠悠，青枫浦上不盛愁。

XU Yuan-chong: Away, away is sailing a single cloud white;

On Farewell Beach are pining away maples green.

ZHAO Yan-chun: Away, away floats a wisp of cloud white;

On the Green Maple shoal I feel so sad.

“青枫浦” is a place name, and it is located in Liuyang county, Hunan Province. Here it means the place that wanderer lived in temporarily.

谁家今夜扁舟子? 何处相思明月楼?

XU Yuan-chong: Where is the wanderer sailing his boat tonight.

Who, pining away, on the moonlit rails would lean?

ZHAO Yan-chun: Who's rowing a canoe against the night?

Who's by a moonlit rail missing her lad?

Here the translation strategies the two translators choose are different, but gained equal effects. “扁舟子” refers to wanderer who row a canoe. “明月楼” is the women's boudoir, here refers to the woman.

斜月沉沉藏海雾, 碣石潇湘无限路。

XU Yuan-chong: In the mist on the sea the slanting moon will hide;

It's a long way from northern hills to southern streams.

ZHAO Yan-chun: The slanting moon looms amid the sea brume;

From him to her stretches an endless way.

The conceptual meaning of “斜月” is “slanting moon”. “碣石”, the name of the mountain, is on the Bohai Sea. “潚 湘" refers to Xiangjiang and Xiaoshui, both belongs to Hunan Province. The two names here, the former in the north and the latter in the south, allude to the long distance between the man and the woman and they are therefore hopelessness of meeting each other.

\section{Application Connotative Meaning to the Translation of Cultural Image Words in Chun Jiang Hua Yue Ye}

Cultural image words include political systems, social customs, historical evolution, traditional etiquette, etc. In addition, there are some words in the language that do not have any emotional color, but because people have long used the words in some social environments, thus it gradually has a deep meaning with special emotional colors (YI Zhong-tian, 2018).

鸿雁长飞光不度, 鱼龙潜跃水成文。

XU Yuan-chong: But message-hearing swans can't fly out of moonlight,

Nor can letter-sending fish can leap out of their place.

ZHAO Yan-chun: But wild geese can never outfly the light,

Nor can fish leap over the ocean's brim.

In this sentence, the author describes that the wild geese are good at flying, unable to fly out of the boundless moonlight to express the sorrowful feeling of the man and the woman because they cannot reach meet each other. The swan goose is a big migratory bird. It comes to the North in spring and goes to the South in autumn. Therefore, it often bows to wanderers, nostalgic for family and sentimental travelers. As early as in the Book of Songs, “鸿雁” was used as a metaphor for the messenger who passed letters between lovers, and it has been used today. In western countries, the wild goose is not common, let alone any sentimental color, and even has any special name. On the contrary, it is called "wild goose". So we can see here XU's version better conveys the meaning of this cultural image word and is acceptable for foreign readers.

In addition, the image of "龙” is completely different in Chinese and Western cultures. In Chinese culture, the dragon is a symbol of sacredness, dignity and auspiciousness. The ancient emperor was called the emperor of the true dragon, and the Chinese were also regarded as the descendants of the dragon. In Western culture, due to the baptism of Christianity, people think that the dragon is the incarnation of the devil Satan, the root of evil, and the symbol of evil and ugliness, so it should be avoided as much as possible. Thus the two versions selected to not translate “龙”.

\section{CONCLUSIONS}


Images are the essence of poetry, because they can arouse the resonance of readers by creating a special scene. Therefore, when translating the cultural image words in Chinese classical poetry, the translators must consider how to express the same emotions in other languages and convey the image of poetry.

Under the guidance of Conceptual Meaning and Connotative Meaning, the author analyzed the English versions of XU Yuan-chong and ZHAO Yan-chun's Chun Jiang Hua Yue Ye, and found that there are only a few culturally image words that can be translated with equivalent expressions in the other languages, while many other kinds cannot for the reason of cultural difference.

From XU Yuan-chong and ZHAO Yan-chun's English version of Chun Jiang Hua Yue Ye, it can be concluded that translators can adopt different strategies in translating cultural image words of Chinese classical poetry, such as literal translation, literal translation plus annotation, free translation, free translation plus annotation, transliteration, and transliteration plus annotation and so on. However, they must remember that the purpose of translation is to fuel communication, so they should pursue to make target language acceptable while attempting to maintain its cultural features of original language in the translation of cultural image words.

\section{REFERENCES}

[1] CHENG Fang-xia \& XU Wen-ting. (2018). A Contrastive Analysis of Chun Jiang Hua Yue Ye and Its English Versions under Theme-Rheme Theory. Journal of Changchun University of Sciences and Technology (Social Sciences Edition), 2, 132-136.

[2] CUI Xuan. (2015). A Study of Cohesive Means in English Versions of Chun Jiang Hua Yue Ye. Journal of Mudanjiang University, 10, 129-131.

[3] HAN Zhong-qian. (2009). From Semantics to Pragmatics: The Development and Influence of Grice's Theory of Meaning. Journal of Anhui University (Philosophy and Social Sciences), 2, 49-54.

[4] HE Yuan-xiu. (2009). The Choices of Senses in Translation from a Modern Semiotics Perspective. Foreign Language and Literature, 2, 140-144.

[5] Hurford, J. R. et al. (2007). Semantics: A Coursebook (Second Edition). Cambridge: Cambridge University Press.

[6] JIA Yan-de. (1992). The Development of Semantic Research. Language Planning, 3, 30-31.

[7] Leech, G. (1974). Semantics. London: Penguin, 1974.

[8] LI Qing-yun. (2013). The Interpretation of ZHANG Ruo-xu's Chun Jiang Hua Yue Ye. Journal of Ezhou University, 3, $31-33$.

[9] LI Shan. (2019). Semantic Selection in Translation from Leech's Semantics Perspective: Taking Chinese-Korean Translation as an Example. Foreign Language Research in Northeast Asia, 2, 90-95.

[10] LI Xiang-de \& HUA Han-Jun. (1990). Semantic Views of Translation. Shanghai Journal of Translators, 3, 7-11.

[11] LUO Shunjiang \& WANG Song. (2012). Three Orientations in Poetry Translation from Eco-translatological Perspective. Journal of Chongqing Jiaotong University (Social Sciences Edition), 5, 123-126.

[12] LÜ Zhaofang \& LIU Junping. (2015). Translation of "The Moon Over the River on a Spring Night" Based on Principle of "Three Beauties". Foreign Language and Literature, 4, 91-95.

[13] Read, A. W. (2015). An Account of the Word 'Semantics'. London \& New York: Routledge, 4, 78-97.

[14] REN Jing. (2015). On the English Translation of Cultural-loaded Words in Chun Jiang Hua Yue Ye from the Perspective of Translation Characteristics. Journal of Lanzhou Institute of Education, 10, 142-143.

[15] Riemer, N. (2016). The Routledge Handbook of Semantics. London \& New York: Routledge.

[16] SHI Bai-li. (2005). Semantics and Translation. Journal of Heilongjiang College of Education, 6, 63-64.

[17] UN Xiao-yan. (2018). A Cognitive Analysis of Nominal Metaphor in Chun Jiang Hua Yue Ye and its English Versions. Journal of Anyang Normal University, 6, 88-90.

[18] TANG Jun \& HE Ting-ting. (2019). A Contrastive Study of English Translation of Images in Chun Jiang Hua Yue Ye from the Perspective of Conceptual Metaphor. Journal of Huaibei Normal University (Philosophy and Social Sciences), 1, 71-75.

[19] WANG Dongfeng. (2005). Rethinking the Translating of Meaning. Foreign Languages in China, 1, 71-78.

[20] WANG Shuang-mei. (2009). Research on the Development Track of Semantics and Its Lagging Reasons. Study \& Exploration, 3, 190-192.

[21] WANG Xiao-ying. (2010). Narrative Perspective and the Loss of Cultural Meaning in the English Translation of Chinese Classical Poetry. Foreign Language Research, 4, 128-131.

[22] WU Qian-guang. (1992). Introduction to Semantics. Changsha: Hunan Education Publishing House.

[23] XU Yuan-chong. (2006). 300 Tang Poems. Beijing: China Translation \& Publishing Corporation.

[24] YAN Ci. (2020). The Translation of Culturally-loaded Words in Chinese Ancient Poetry: Taking ZHAO Yan-chun's English Translation of Chun Jiang Hua Yue Ye as an Example. Chinese \& Foreign Entrepreneurs, 7, 237-239.

[25] YI Zhong-tian. (2018). Reading Poetry of Dang Dynasty. Shanghai: Shanghai Literature and Art Publishing House.

[26] ZENG Xiancai. (1993). Semantic, Pragmatic and Translation. Modern Foreign Languages, 1, 23-27.

[27] ZHANG Jing-hao. (2000). A Pen Talk on Translation with Nida. Chinese Translators Journal, 5, 28-33.

[28] ZHANG Tong-tong. (2016). A Comparative Analysis of Two English Versions of Chun Jiang Hua Yue Ye_from the Perspective of Relevance Theory. Journal of Language and Literature Studies, 3, 77-79.

Peng Wang, studies at School of Foreign Languages, Guizhou Normal University. His research interests lie in the fields of translation and cross-culture studies. Email: WP12151215@163.com 